\title{
A combination of cranial and peripheral nerve palsies in infectious mononucleosis
}

\author{
K. Mohanaruban and D.J.H. Fisher \\ Department of Medicine, Cardiff Royal Infirmary, Newport Road, Cardiff CF2 1SZ, UK.
}

\begin{abstract}
Summary: A 44 year old woman presented with bilateral brachial neuritis and an isolated Bell's palsy. Subsequently she was found to have infectious mononucleosis. The association of cranial and brachial neuropathy has not previously been reported. Without any specific drug therapy she recovered completely within 5 months.
\end{abstract}

\section{Introduction}

Neurological complications from infectious mononucleosis, although well recognized, are rare. Their incidence has been recorded as between $0.37 \%$ to $7.3 \%$, although changes in the cerebrospinal fluid are reported to occur in about $26.5 \%$ of cases (Silverstein et al., 1972). The more common neurological complications include meningitis, encephalitis, GuillainBarré syndrome, transverse myelitis, peripheral neuropathy, cranial neuropathies, acute cerebellar ataxias, as well as psychological illnesses. Isolated brachial plexus involvement is a rare complication (Radin, 1967; Liveson \& Goodgold, 1974; Watson \& Ashby, 1976) and we believe the case we are reporting to be the first patient with infectious mononucleosis presenting with both bilateral brachial neuritis and Bell's palsy.

\section{Case report}

A 44 year old woman developed a sore throat, fever, night sweats and general malaise. Approximately 7 days later, she developed severe pain around both shoulder girdles, mainly over the deltoid muscles, followed a few days later with weakness of her left arm with some sensory disturbance in the same limb. Twenty-four hours later, she developed weakness of the right arm, but no sensory disturbance. The day before admission, 3 weeks after the initial symptoms of her illness, her husband noted asymmetry of the left side of her face.

On clinical examination the patient did not have pharyngitis, rash, lymphadenopathy, or hepatosplenomegaly. Cranial nerve examination showed a left

Correspondence: K. Mohanaruban M.R.C..S., M.R.C.P. Accepted: 20 June 1986 lower motor neurone seventh nerve palsy; the rest of the cranial nerves were intact. There was moderate weakness of both deltoid muscles, marked weakness of the left infraspinatus muscle and obvious bilateral winging of scapulae, the left side being more prominent than the right. There were no sensory abnormalities and the remainder of the neurological examination was normal.

\section{Investigations}

Haemoglobin $11.5 \mathrm{~g} / \mathrm{dl}$, white cell count $6.5 / 1$ (neutrophils $50 \%$, lymphocytes $39 \%$, monocytes $6 \%$, eosinophils $4 \%$ ), platelets $410 \times 10^{9} / 1$. The blood picture was reported as normal apart from reactive lymphocytes. The serum alkaline phosphatase and aspartate transaminase levels were slightly raised. The Paul Bunnell test titres were: after incubating with sheep red cells 640, guinea pig kidney 640, and ox red cells 10 . After seven days the respective titres were 1,200,1,200 and 10. Cerebrospinal fluid examination showed normal protein levels and 'very scanty polymorphs'.

During her stay in hospital she was treated with simple analgesia, bedrest and physiotherapy. Her symptoms and signs gradually improved. She was discharged home after 3 weeks and over the course of the next 5 months she made a slow and complete recovery from all her neurological deficits.

\section{Discussion}

Brachial plexus neuropathy is unusual in infectious mononucleosis, and in a large series of 99 patients presenting with brachial plexus neuropathy (Tsairis $e t$ al., 1972) only two were diagnosed as due to infectious

(C) The Fellowship of Postgraduate Medicine, 1986 
mononucleosis. We present this case to illustrate that the neurological manifestations of infectious mononucleosis can involve both cranial and peripheral nerves

\section{References}

LIVESON, J.A. \& GOODGOLD, J. (1974). Mononeuritis multiplex associated with infectious mononucleosis. Canadian Journal of Neurological Sciences, 1, 203.

RADIN, E.L. (1967). Peripheral neuritis as a complication of infectious mononucleosis. Journal of Bone and Joint Surgery, 49, 535.

SILVERSTEIN, A., STEINBERG, G. \& NATHANSON, M. (1972). Nervous system involvement in infectious mono- at the same time, and may not be merely confined to one nerve root. The complete recovery in our patient is in keeping with the reported experience of others.

nuclensis. Archives of Neurology, 26, 353.

TSAIRIS, P., DYCK, P.J. \& MULDER, D.W. (1972). Natural history of brachial plexus neuropathy. Archives of Neurology, 27, 109.

WATSON, P. \& ASHBY, P. (1976). Brachial plexus neuropathy associated with infectious mononucleosis. Canadian Medical Association Journal, 114, 758. 